\title{
Deciphering bifidobacterial-mediated metabolic interactions and their impact on gut microbiota by a multi-omics approach
}

\author{
Francesca Turroni ${ }^{1,2}$, Christian Milani ${ }^{2}$, Sabrina Duranti ${ }^{2}$, Leonardo Mancabelli ${ }^{2}$, \\ Marta Mangifesta ${ }^{3}$, Alice Viappiani ${ }^{3}$, Gabriele Andrea Lugli², Chiara Ferrario ${ }^{2}$, \\ Laura Gioiosa ${ }^{4}$, Alberto Ferrarini ${ }^{5}$, Jia Li ${ }^{6}$, Paola Palanza ${ }^{4}$, Massimo Delledonne ${ }^{5}$, \\ Douwe van Sinderen ${ }^{1}$ and Marco Ventura ${ }^{2}$ \\ ${ }^{1}$ APC Microbiome Institute and School of Microbiology, National University of Ireland, Cork, Ireland; \\ ${ }^{2}$ Laboratory of Probiogenomics, Department of Life Sciences, University of Parma, Parma, Italy; ${ }^{3}$ GenProbio \\ srl, Parma, Italy; ${ }^{4}$ Department of Neurosciences, University of Parma, Parma, Italy; ${ }^{5}$ Department of \\ Biotechnology, University of Verona, Verona, Italy and ${ }^{6}$ Department of Surgery and Cancer, Faculty of \\ Medicine, Imperial College, London, UK
}

\begin{abstract}
The intricacies of cooperation and competition between microorganisms are poorly investigated for particular components of the gut microbiota. In order to obtain insights into the manner by which different bifidobacterial species coexist in the mammalian gut, we investigated possible interactions between four human gut commensals, Bifidobacterium bifidum PRL2010, Bifidobacterium adolescentis 22L, Bifidobacterium breve 12L and Bifidobacterium longum subsp. infantis ATCC15697, in the intestine of conventional mice. The generated information revealed various ecological/metabolic strategies, including glycan-harvesting, glycan-breakdown and cross-feeding behavior, adopted by bifidobacteria in the highly competitive environment of the mammalian intestine. Introduction of two or multiple bifidobacterial strains caused a clear shift in the microbiota composition of the murine cecum. Whole-genome transcription profiling coupled with metagenomic analyses of single, dual or multiple associations of bifidobacterial strains revealed an expansion of the murine gut glycobiome toward enzymatic degradation of plant-derived carbohydrates, such as xylan, arabinoxylan, starch and host-derived glycan substrates. Furthermore, these bifidobacterial communities evoked major changes in the metabolomic profile of the microbiota as observed by shifts in short chain fatty acid production and carbohydrate availability in the murine cecum. Overall, these data support an ecological role of bifidobacteria acting directly or through cross-feeding activities in shaping the gut murine microbiome to instigate an enrichment of saccharolytic microbiota.
\end{abstract}

The ISME Journal (2016) 10, 1656-1668; doi:10.1038/ismej.2015.236; published online 9 February 2016

\section{Introduction}

The interactions between bacteria residing in the human gut influence the health status of the host by modifying human metabolism and affecting the presence and virulence of pathogens (Robles Alonso and Guarner, 2013; Rescigno, 2014). In fact, the relationships that exist between members of the gut microbiota may involve synergistic actions (between autochthonous components of gut microbiota) or antagonistic events, like those occurring between transient and indigenous gut microbiota

Correspondence: M Ventura, Laboratory of Probiogenomics, Department of Life Sciences, University of Parma, Parco Area delle Scienze, 11/a, Parma 43124, Italy.

E-mail: marco.ventura@unipr.it

Received 23 July 2015; revised 12 October 2015; accepted 10 November 2015; published online 9 February 2016 members (Sonnenburg et al., 2004). Bifidobacteria represent prominent commensals of the human infant gut (Turroni et al., 2012a), where they are believed to modulate metabolic and immune activities of their host (Macfarlane et al., 2011; Ventura et al., 2012). Furthermore, cross-feeding strategies through expansion of carbohydrate acquisition abilities may be used by gut bacteria, thereby positively influencing their ecological fitness (Tannock et al., 2012; Egan et al., 2014a, b). Cross-feeding represents an ecological strategy whereby for example dietary or host-derived polysaccharides are extracellulary hydrolyzed in the mammalian gut by enteric microorganisms such as bifidobacteria to produce simpler glycans (monosaccharides and oligosaccharides) that then become available to other microbial gut inhabitants (De Vuyst and Leroy, 2011). Following the fermentative metabolism of such glycans the 
end-metabolites (in particular acetate) may serve as substrates for secondary degraders such as butyrate-producing colonic bacteria (Barcenilla et al., 2000; Morrison et al., 2006; Duncan and Flint, 2008; Falony et al., 2009; Munoz-Tamayo et al., 2011). Through functional genomic approaches, significant progress has been made toward unraveling bifidobacterial gut persistence strategies and discovery of microbe-host interactions (Sonnenburg et al., 2006; Ventura et al., 2009). Recently, genome analyses of the human gut bifidobacteria Bifidobacterium bifidum PRL2010, Bifidobacterium adolescentis 22L, Bifidobacterium breve 12L and Bifidobacterium longum subsp. infantis ATCC15697 clearly support the notion of extensive genetic adaptations to facilitate life in the human gut including their ability to utilize host-produced glycans such as mucin and human milk oligosaccharides, or to access dietary complex carbohydrates like starch (Sela et al., 2008; Turroni et al., 2010; Bottacini et al., 2014; Duranti et al., 2014). Here, we describe the molecular impact established under in vivo conditions between the above-mentioned set of bifidobacteria. We show that coexistence of these bifidobacterial strains influences their saccharolytic phenotype, whereas their presence also impacts on the murine cecal microbiota and metabolome.

\section{Materials and methods}

Bifidobacteria were manipulated and used as described in Supplementary Materials and methods. Detailed descriptions of murine trials and methods for gene expression analyses, $16 \mathrm{~S}$ ribosomal RNA (rRNA) microbial profiling experiments, shotgun metagenomic investigations and metabolomic assays are provided in Supplementary Materials and methods. The transcriptional array data have been deposited in the GEO database under accession number GSE65829. The 16S rRNA microbial profiling and shotgun metagenomic data sets have been deposited under accession numbers SRP052936 and SRP052737, respectively. RNAseq raw data from this study were deposited in the SRA database under accession number SRP061182.

\section{Results and Discussion}

Bifidobacterial presence in the murine gut Eleven groups of five conventional female 8-week-old $\mathrm{BALB} / \mathrm{c}$ mice, being fed a standard polysacchariderich Chow diet, were for 5 days administered a single daily dose of $10^{9}$ colony forming unit of either B. bifidum PRL2010, B. breve 12L (Bottacini et al., 2014), B. adolescentis 22L (Duranti et al., 2014), B. longum subsp. infantis ATCC15697 (Sela et al., 2008) or bifidobacterial couples, that is, PRL201012L, PRL2010-22L, PRL2010-ATCC15696, 12L-22L, 12L-ATCC15697, 22L-ATCC15697, or a combination of all four bifidobacterial strains (Supplementary Information). These bifidobacterial strains had all been isolated from a common ecological niche, that is, the human neonatal gut (Reuter, 1963; Turroni et al., 2010; Bottacini et al., 2014; Duranti et al., 2014), thus implying a natural predisposition to interact. Mice were $a$ priori checked for the presence of bifidobacteria in fecal samples by PCR using Bifidobacterium-specific primers (Turroni et al., 2009), revealing that bifidobacteria were either absent or below the limit of detection $\left(<10 \mathrm{E} 1 \mathrm{~g}^{-1}\right.$ of fecal material). In addition, the native murine microbiota was investigated in depth by means of a shotgun metagenomics approach of all animals before the administration of bifidobacteria (the average number of paired-end reads for sample >5.7E6). The obtained murine fecal microbiome was mapped on a set of bifidobacterial genes that had previously been shown to allow for a precise detection of bifidobacteria (Ferrario et al., 2015) involving the core-genome sequences of the genus Bifidobacterium (Milani et al., 2014). Consistent with the PCR findings, even at $85 \%$ identity cutoff, no reads of the obtained T0 data set (murine sample obtained before bifidobacterial administration, Supplementary Figure S1) were shown to map on to this bifidobacterial core-genome, thus indicating the absence of bifidobacteria in the untreated mice (Supplementary Table S1). As expected, when we mapped the core-genome sequences of the genus Bifidobacterium (Milani et al., 2014) on the fecal microbiome from mice receiving any combination of the four bifidobacterial strains at T2 (murine sample obtained after 5 days of bifidobacterial administration, Supplementary Figure S1), we readily identified bifidobacterial reads at $100 \%$ nucleotide identity (Supplementary Table S1). Analysis of murine fecal samples for the presence of native bifidobacterial DNA using quantitative PCR approaches involving bifidobacterial-specific primers (Matsuki et al., 2002; Gueimonde et al., 2004) also failed to detect bifidobacteria. These findings were further confirmed by traditional microbiological methods, that is, plating on bifidobacterial selective medium. The absence of bifidobacteria in murine fecal samples has been described previously for other in vivo studies involving conventional adult $\mathrm{BALB} / \mathrm{c}$ mice (O'Connell Motherway et al., 2011; Turroni et al., 2013, 2014). Bifidobacteria have nevertheless been detected in stool samples of mice in one study (Zhang et al., 2013), but their presence may be dependent on a number of host factors (for example, the murine breedand/or age of the animals) and/or environmental factors (for example, the type of diet, supplier and conditions of housing systems). Overall, based on a cultivation approach, PCRbased approaches and shotgun metagenomic attempts, we conclude that no bifidobacteria were present at detectable levels in the murine gut microbiota before bifidobacterial administration. 
Animals were killed 12 days after the first bifidobacterial administration, allowing sufficient time for several cycles of turnover of the intestinal epithelium and its overlying mucus layer (Wolfensohn and Lloyd, 2003). Quantitative evaluation of bifidobacteria present in the murine gut upon bacterial supplementation was assessed using strainspecific primers through real-time quantitative PCR (Supplementary Information). Such data demonstrated that each of the administered bifidobacterial strains were present at stable numbers at time points after cessation of bifidobacterial administration (T3 and T4), reminiscent of at least transient presence, with the highest bifidobacterial numbers recorded in the cecum (Supplementary Figure S1). Microbial density was not substantially different among any of the strains when they were introduced on their own $\left(10^{4}-10^{5} \mathrm{log}\right.$; genome copy number $\mathrm{g}^{-1}$ mouse feces). Interestingly, the co-occurrence of all four bifidobacterial strains in the murine cecum (multiple association) provokes an increased level of each bifidobacterial strain compared with the respective strain population size in mono-association. This observation suggests that specific cross-feeding activities exist between some of the bifidobacterial populations analyzed in the murine gut, apparently allowing improved nutrient harvesting thereby causing an overall higher level of these, at least transiently present, bifidobacteria. Given their (transient) presence in the distal murine gut, we examined possible metabolic interactions between the various combinations of bifidobacterial strains in this ecological niche.

\section{The transcriptome of bifidobacterial strains under} in vivo conditions

To gain a genome-wide view of possible effects of bifidobacterial co-association on each strain's transcriptome, RNA was extracted from the cecal contents of mice in each treatment group. The transcripts expressed in B. bifidum PRL2010, $B$. breve $12 \mathrm{~L}, B$. adolescentis $22 \mathrm{~L}$ and $B$. longum subsp. infantis ATCC15697 were profiled using a custom-made PRL2010-12L-22L-ATCC15697 (multibifido)-array representing 100\% (PRL2010), 99\% (12L and ATCC15697) and 96\% (22L) of the identified genes of these organisms. Probe pairs were designed in a way so as to minimize crosshybridization between genes contained within and between bifidobacterial genomes, which was verified experimentally by hybridizing the multibifido-array to complementary DNA targets prepared from animals administered a single bacterial species (monoassociation). In addition, in order to evaluate putative cross-hybridization of the microarray probes with related (action)bacteria present in the murine fecal samples, we performed mapping of the 60 nt-long DNA sequences of the microarray probes on the fecal microbiome data achieved for each mouse before bifidobacterial administration (T0).
Notably, at $85 \%$ nucleotide identity cutoff, which was identical to that used in microarray hybridization experiments, only $0.11 \%$ of the probes produced detectable hybridization signals (Supplementary Table S2). Interestingly, these probes encompass 32 genes (of a total of 7603) predominantly encoding housekeeping functions, which are highly conserved among bacterial genera. These data confirm that the multibifido microarray was highly specific for the PRL2010-12L-22LATCC15697 strains. The specificity of the experimental set-up and lack of spurious signals was confirmed by hybridizing the multibifido array with fecal DNA extracted from a mix of all T0 samples (Supplementary Figure S1). The obtained transcriptome data were confirmed by real-time quantitative PCR involving key functional genes identified from microarray-based analyses (Supplementary Table S3). A variable number of genes, ranging from 16 to 137 , exhibited a $\geqslant 2$-fold change $(P<0.001)$ in transcription upon the introduction of different bifidobacterial strains in the murine cecum (bi- or multi-association), using the transcriptome of each cecal mono-association as a reference condition (Supplementary Figure S1). Functional categorization of these differentially expressed genes using cluster orthologues gene revealed a metabolism-biased response to co-association including statistically significant over-representation of upregulated PRL2010, 12L, 22L, ATCC15697 genes involved in carbohydrate utilization, which suggests that these microorganisms utilize such carbohydrates for their own metabolic purposes (Figure 1, Supplementary Figure S2). Furthermore, a significant impact on amino-acid metabolism was evident in transcriptomes of bi-associations. Notably, we observed that the $B$. breve 12L and B. bifidum PRL2010 transcriptomes were most substantially changed, affecting $13.2 \%$ and $12 \%$ of examined genes, respectively (cutoff values of $\geqslant 2$-fold change $(P<0.001)$ and $\leqslant 0.5$ $(P<0.001))$, by the bi-association with another bifidobacterial strain, suggesting that $12 \mathrm{~L}$ and PRL2010 are most affected by such interactions (Figure 1).

\section{Co-association prompts bifidobacterial utilization of carbohydrates}

When we examined the transcriptomes of the cecal bi-associations we observed a significant transcriptional increase $(>2$-fold induction and $P<0.001)$ of several genes encoding predicted glycoside hydrolases (GHs) according to the CAZy database (Lombard et al., 2014), as well as of genes predicted to encode carriers dedicated to carbohydrate transport. Remarkably, among the different bifidobacterial strains investigated here, transcription of carbohydrate metabolism-related genes from $B$. adolescentis $22 \mathrm{~L}$ was shown to be most severely affected by co-association, followed by those of B. bifidum 

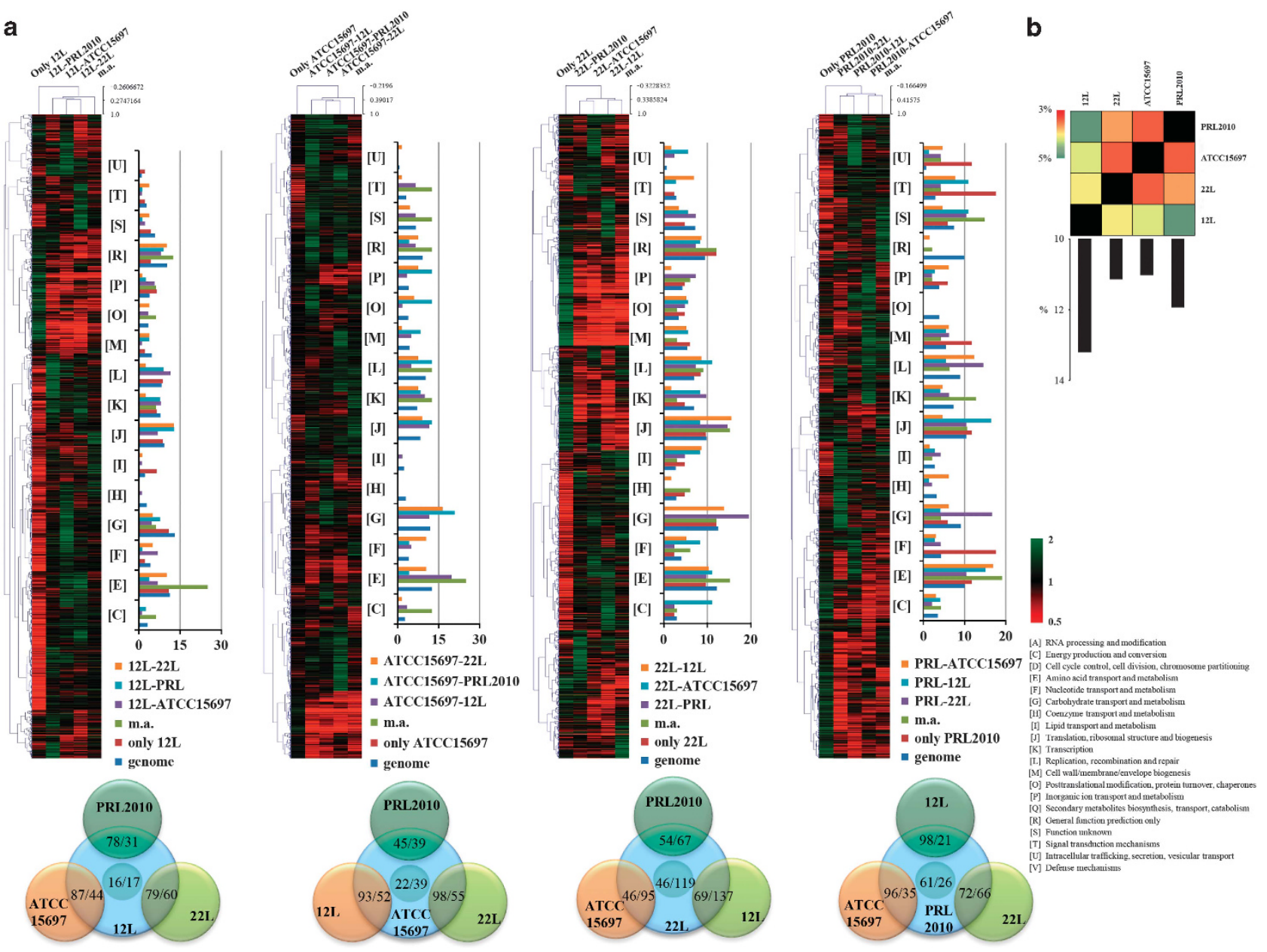

Figure 1 Transcriptomes of bifidobacteria when present in the murine cecum. (a) Identification of B. breve 12L, B. longum subsp. infantis ATCC15697, B. adolescentis 22L and B. bifidum PRL2010 differentially expressed genes by transcriptome analysis in response to monobi- or multiple association (m.a.) in mice. Each heat map displays the fold change in gene expression for 12L, ATCC15697, 22L and PRL2010 (from right to left) according to the condition indicated above the heat map. Each row represents a separate transcript and each column represents a separate sample. A color legend is displayed on the bottom of the microarray plot. The dendrogram on the left margin of the heat map represents the hierarchical clustering algorithm result based on average linkage (UPGMA) and Euclidean distance of the gene data set. Functional annotation of the in vivo-expressed genes of the different bifidobacterial strains according to their cluster orthologues gene (COG) categories is shown at the right-hand margin of each heat map. Each COG family is identified by a one-letter abbreviation (NCBI database). The percentage was calculated as the percentage of transcribed genes belonging to the indicated COG category with respect to all transcribed genes. A Venn diagram displaying the number of genes expressed by 12L, ATCC15697, 22L and PRL2010 strain upon contact each other is represented at the bottom of each heat map. (b) A heat map representing the cross-talk index. Bar plots represent the sum of the single cross-talk indexes for each strain. Values are expressed as percentages.

PRL2010. In fact, we observed that transcription of $26.3 \%$ and $22.7 \%$ of the total GH-specifying genetic arsenal was shown to be affected when $B$. adolescentis 22L and B. bifidum PRL2010, respectively, were in cecal co-association with other bifidobacteria. In this context, B. adolescentis 22L showed significant changes ( $>2$-fold induction and $P<0.001)$ in the transcription of genes encoding GHs belonging to the GH3, GH43 and GH53 families, members of which are known to be involved in the metabolism of plant cell wall glycans, such as arabinoxylan and arabinogalactan (Lombard et al., 2014). B. bifidum PRL2010 displayed transcriptional changes corresponding to genes encoding GH13 and GH51 members, which are involved in the breakdown of plant-related polysaccharides such as starch and xylan (Lombard et al., 2014). Transcriptional increase of genes that are predicted to be dedicated to hydrolysis of xylose-containing glycans was observed in almost all bifidobacterial strains when they co-occur in the cecum (bi- and multiple associations). Interestingly, when $B$. bifidum PRL2010 was in cecal association with $B$. adolescentis 22L, the latter strain exhibited transcriptional increase of three genes predicted to encode xylosidedegrading enzymes belonging to the GH43 family (BADO_0433, BADO_0453, BADO_1485) (NB. a fourth member of this GH family, BADO_0164, seems to be constitutively transcribed). A putative acetylxylan esterase-encoding gene (BADO_1473), 
presumed to be responsible for xylan deacetylation (Poutanen et al., 1990), was shown to be transcribed under all conditions tested, whereas increased transcription of two putative $\alpha$-L-arabinofuranosidase-encoding genes (BADO_0428 and BADO_1487) was observed when $22 \mathrm{~L}$ is present in cecal biassociation with $B$. bifidum PRL2010. In contrast, the endo-1,4- $\beta$-xylanase-specifying gene (BBPR_0379) of B. bifidum PRL2010 was shown to be transcribed when in mono-association, in bi-association with $22 \mathrm{~L}$ or in multiple association with the other three bifidobacterial strains. $B$. longum subsp. infantis ATCC15697 possesses a predicted endo-1,4- $\beta$-xylanase-encoding gene (Blon_2411), which was shown to be transcribed at low or below background level when ATCC15697 is in cecal multi-association (Supplementary Figure S2). Notably, predicted xylose-liberating hydrolases have previously been shown to be subject to upregulation during co-association of Bifidobacterium longum subsp. longum NCC503 and Bacteroides thetaiotaomicron in the cecum of otherwise germ-free mice (Sonnenburg et al., 2006). These findings suggest that xylose metabolism becomes enhanced when bifidobacteria have to coexist with other, carbohydrate-competing gut microorganisms. The transcriptional upregulation of genes involved in the degradation and metabolism of xylose-containing poly/oligosaccharides by almost all bifidobacterial strains when arranged in bi- or multiple association may be a result of bifidobacterial strains turning their metabolic attention to an available, but less-coveted substrate that is ignored in mono-associations, but accessed when preferred substrates become (more quickly) depleted in co-association. Although less parsimonious, an alternative possibility is that each strain, when present in mono-association in the cecum, possesses a complement of GHs that is insufficient for efficient degradation of available xylose-containing glycans. However, when their repertoires of GHs are combined, complementary enzyme activities allow these bifidobacterial strains to participate in a synergistic harvest of this glycan type, in a manner, which is similar to that previously observed for cellulose-metabolizing bacterial communities (Fondevila and Dehority, 1994; Kato et al., 2005). Furthermore, we reported transcriptional upregulation, especially in multiassociation or in the bi-association of $B$. adolescentis 22L and $B$. breve 12L, of genes encoding enzymes belonging to the GH 43 family, known to be involved in starch degradation. These latter two strains possess the entire genetic arsenal required for the complete breakdown of starch (Duranti et al., 2014), although only 22L is predicted to encode three extracellular alpha-amylases (BADO_0753, BADO_1638, BADO_1639), one intracellular alpha-amylase (BADO_1572) and one extracellular pullulanase (BADO_0754) (Supplementary Figure S2). The alpha-amylase-encoding gene BADO_1572 was shown to be highly expressed under all conditions tested. In contrast, the extracellular $\alpha$-amylase-encoding gene BADO_1639 exhibited increased transcription (ranging from 2. 6- to 4-fold expression, $P<0.001$ ) when $22 \mathrm{~L}$ in bi-association with $B$. bifidum PRL2010 or with B. longum subsp. infantis ATCC15697. Analyzing the transcriptome of $B$. longum subsp. infantis ATCC15697, we observed an upregulation of two genes encoding a putative 4- $\alpha$-glucanotranferase (Blon_0139) and phosphoglucomutase (Blon_2184), especially when this strain was present with $B$. adolescentis 22L. These enzymes are likely to act on maltodextrin and maltose residues, which represent end products of $\alpha$-amylase and pullulanase activities.

We furthermore evaluated the average and co-association-specific transcription levels of $B$. breve 12L, B. adolescentis 22L, B. bifidum PRL2010 and B. longum subsp. infantis ATCC15697 genes encoding enzymes involved in simple sugar utilization, in particular sugars that are commonly detected in the murine cecum, such as arabinose, xylose, galactose and aminosugars (Sonnenburg et al., 2006; Supplementary Figure S3). No significant transcriptional changes were identified for genes associated with arabinose utilization by the investigated bifidobacterial strains (Supplementary Figure S3). In contrast, we observed an upregulation of the majority of genes predicted to be involved in xylose metabolism for the four bifidobacterial strains when they are in bi- or multi-associations, in particular for B. adolescentis 22L and B. bifidum PRL2010, which possess a large repertoire of such genes (Turroni et al., 2010; Duranti et al., 2014). In this context, significant $(P<0.001)$ upregulation was evident for the xylulose kinase-encoding gene (BADO_0347) and phosphoglycerate mutasespecifying gene (BADO_0663), when B. adolescentis $22 \mathrm{~L}$ was present in cecal bi-association with $B$. bifidum PRL2010, whereas the majority of genes linked to xylose metabolism in B. bifidum PRL2010 (16 out of 21 genes) exhibited increased transcription under all associations tested (Supplementary Figure S3). Notably, xylose is the final product of xylan degradation, a process that, as mentioned above, was shown to be enhanced upon co-occurrence of bifidobacterial strains. Transcription of genes associated with galactose metabolism was shown to be induced in B. bifidum PRL2010 for all evaluated associations, and a less pronounced upregulation or the same genetic repertoire was also observed for $B$. breve $12 \mathrm{~L}$ and $B$. longum subsp. infantis ATCC15697, especially when they were in co-association with each other. Transcription of genes putatively involved in hexosamine utilization was shown to be upregulated in B. bifidum PRL2010 and $B$. adolescentis 22L, especially when these two strains were in co-association (Supplementary Figure S3). As previously described, B. bifidum strains target galactose- and hexosamine-containing substrates such as lactose and glycoproteins (Turroni 
et al., 2012b). Evaluation of the transcriptional profiles of genes predicted to be involved in carbohydrate uptake (Sela et al., 2008; Turroni et al., 2012b; Bottacini et al., 2014; Duranti et al., 2014) highlighted varying responsiveness among the evaluated strains. In fact, for PRL2010, 12L and 22L strains, we observed an upregulation of most of their combined carbohydrate transporter-encoding gene repertoire when these bacteria were in co-association, as compared with transcription levels of such genes when in monoassociation (Supplementary Figure S4). This reflects a scenario that was observed for the transcription of genes involved in the metabolism of simple sugars (see above). In contrast, transcription of the predicted carbohydrate transporter-encoded gene set of $B$. longum subsp. infantis ATCC15697 was shown to be unaltered by the presence of other bifidobacterial strains here evaluated (Supplementary Figure S4). This latter result is consistent with the apparently non-interactive behavior of this strain with other bifidobacteria, reinforcing the notion that ATCC15697 may act as a solitary factor in its ecological niche. Nevertheless, we cannot exclude that ATCC15697 interacts with other components of the gut microbiota.

\section{In vitro evaluation of cross-feeding features of} bifidobacteria

In order to confirm the synergistic effects related to the utilization of xylose-containing glycans that appear to occur between the PRL2010-12L-22LATCC15697 strains, we assayed the transcriptomes of these bacteria when co-cultivated under in vitro conditions on MRS containing xylan as the unique carbon source. Transcriptomic data sets recovered from RNAseq experiments involving this multiple strain association cultivated on xylan were compared with the transcriptomes achieved for each mono-association grown on the identical substrate. The transcriptomes of this multiple bifidobacterial strain association grown on xylan clearly pointed out the expression of a sizable number of different GH-encoding genes, such as those predicted to belong to GH43 of B. adolescentis 22L (BADO_0433, BADO_0453 and BADO_1485) as well as accessory genes predicted to be involved in the metabolism of xylose such as the BADO_1473 predicted to encode the acetylxylan esterase (Supplementary Figure S2). In addition, we observed the transcription of Blon_2411 of ATCC15697 that is predicted to specify an endo-1,4- $\beta$-xylanase (Supplementary Figure S2) directed to facilitate the breakdown of xylan-containing carbohydrates. Finally, RNAseq experiments indicated that the complete predicted genetic arsenal of $B$. bifidum PRL2010 strain involved in the metabolism of xylose exhibited increased transcription (Supplementary Figure S2). Altogether, these in vitro data confirmed what was observed under in vivo conditions, although the precise number of identified genes and their induction levels were not identical, which is more than likely due to the influence of a complex cecum murine microbiota in the in vivo trial.

\section{Impact of bifidobacterial presence on the murine microbiota}

The observed functional changes in the examined bifidobacterial transcriptomes may be caused by shifts of the murine cecum microbiota because of the presence of bifidobacteria. Thus, we assessed if the presence of PRL2010, 12L, 22L and ATCC15697 strains in mono-, bi- or multi-association in the murine cecum affects the overall composition of the microbiota of this environment. For this purpose, we analyzed the microbiota composition of cecum samples retrieved from T0 mice (murine fecal samples before the administration of bifidobacteria), representing the reference condition, as well as mono-, bi- and multiple associations at T1, T2, T3 and T4 by means of 16S rRNA gene-based profiling. The extent of the cecal microbial biodiversity was assessed in an exhaustive manner as displayed by the plateauing trend of the rarefaction curves (Supplementary Table S4, Supplementary Figure S5). De novo clustering of de-noised high quality reads generated operation taxonomic units (OTUs) that were taxonomically assigned down to genus level. Taxonomic allocation of these reads at phylum level revealed that Firmicutes represented the dominant phylum, followed by Bacteroidetes, comprising $64 \%$ and $28 \%$, respectively, of the total sequences in tested cecal samples (Supplementary Fig. S6). When the cecum-associated microbiota composition was compared at family level between samples taken at different time points and containing the same bifidobacterial strains (for example, mono-, bi- and multiple association data sets), we observed a time-dependent increase in the relative abundance of Rikenellaceae and a decrease in the relative abundance of Lachnospiraceae, induced by the addition of at least a single bifidobacterial strain (Supplementary Figure S6). We observed also an apparent stimulatory effect of $B$. bifidum PRL2010 on the relative abundance of Bacteroidaceae, especially when PRL2010 was found in bi-association with other bifidobacterial species (Supplementary Figure S6). In addition, the $S 24-7$ family's relative abundance increased when $B$. adolescentis 22L was present in mono-association. Hierarchical cluster representation based on a Pearson correlation matrix built using family-level composition, allowed clustering of analyzed samples into three main groups (Figure 2). Notably, group A encompasses the majority of T0 samples as well as samples from mice that had been administered $B$. longum subsp. infantis ATCC15697, B. breve 12L, or both in bi-association, reminiscent of their weak modulatory effect on the microbiota composition (Figure 2). Group B includes the majority of murine fecal samples that had been administered bifidobacteria (Figure 2). Finally, group C mainly encompasses 


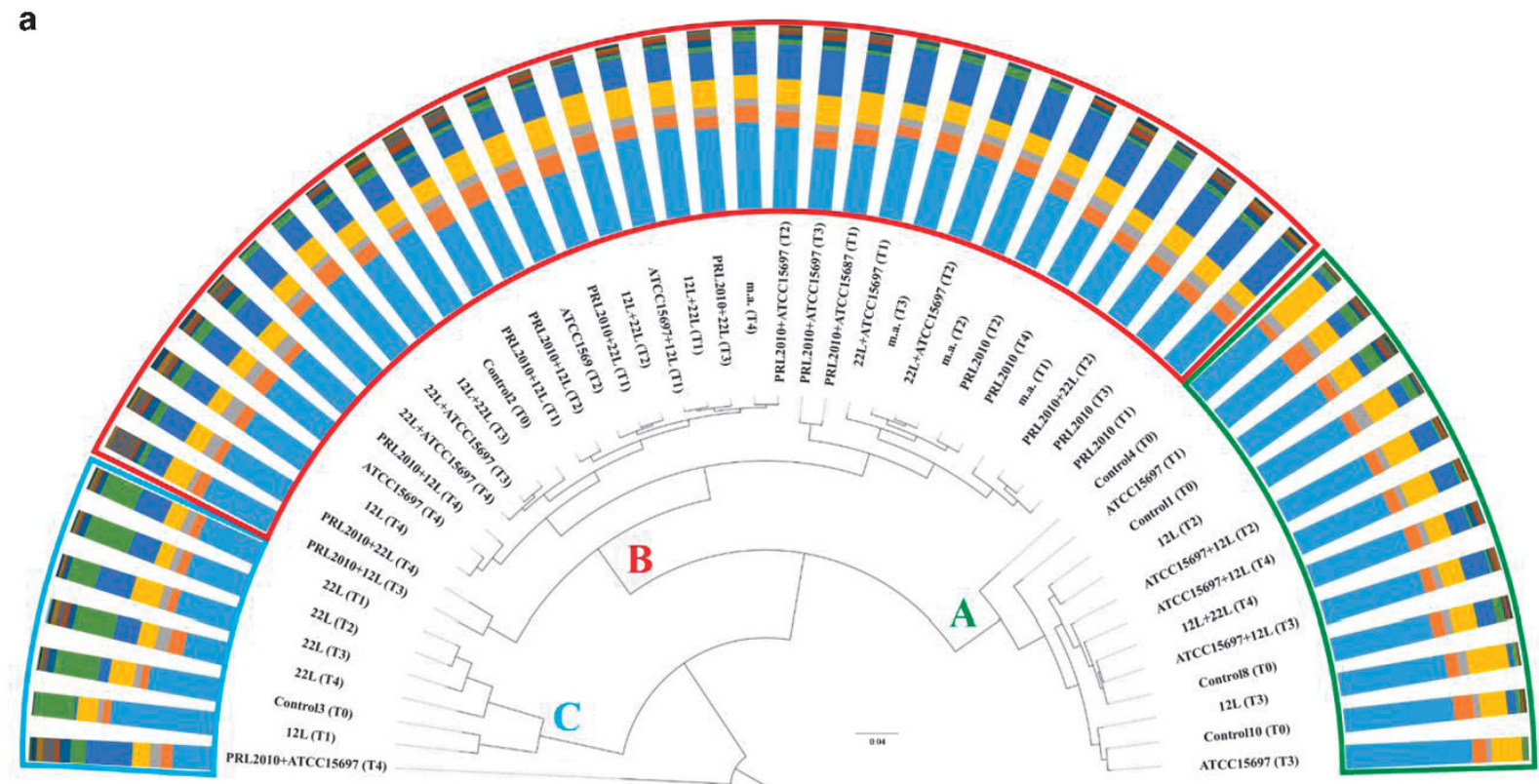

b

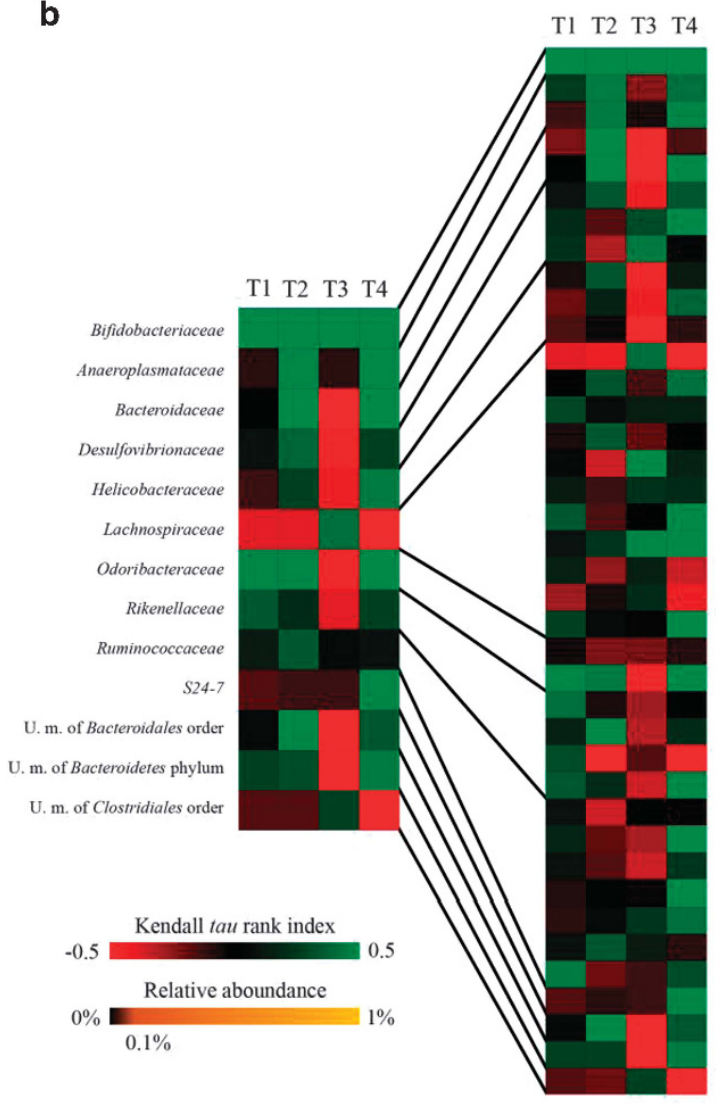

T1 T2 T3 T4

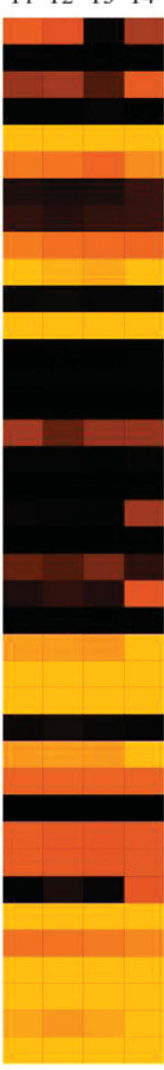

C

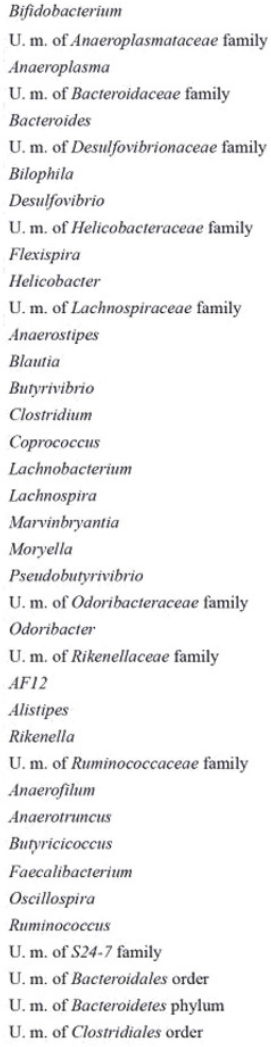

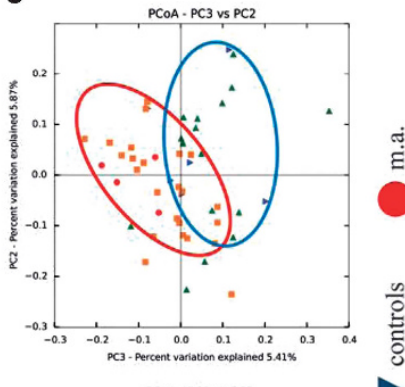
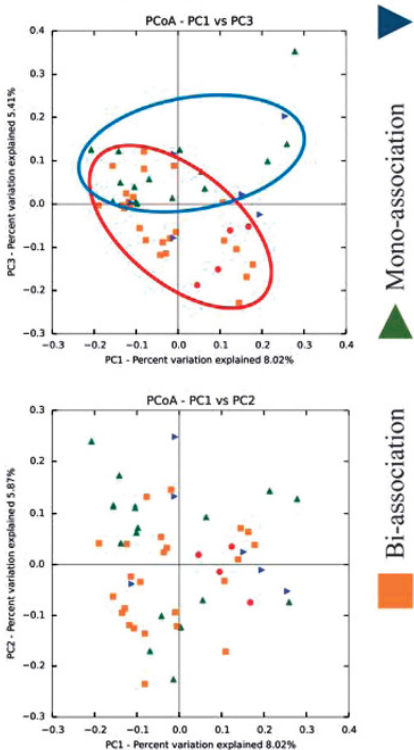

Figure 2 Modulation of murine fecal microbiota composition by the presence of bifidobacteria. (a) Displays the hierarchical clustering of the sample composition at family level based on a Pearson correlation matrix allowed the identification of three main clustering groups, named A, B and C. Microbiota profiling at family level of each sample is shown as a bar plot showing only families with relative abundances $>1 \%$. (b) Shows the Kendall tau rank generated when considering the average 16S rRNA profiles for each time point for the mono-, bi- and multiple associations, with respect to the Bifidobacteriaceae family and Bifidobacterium genus. Only genera constituting families with relative abundance $>1 \%$ are shown. A positive Kendall tau rank value indicates co-occurrence, whereas a negative Kendall tau rank value indicates co-exclusion. (c) Shows the Unifrac beta-diversity expressed as principal coordinate analysis (PCoA) illustrating all the T0 and mono-, bi- and multiple association samples. T0 samples and mono-associations (circled in blue) cluster separately from biand multiple associations (circled in red). 
B. adolescentis 22L mono-association samples that cluster apart as expected, given their prominent stimulatory effect on the relative abundance of the S24-7 family. In order to assess if and to what extent bacterial communities were different between the various examined samples, the significance test in UniFrac (Lozupone et al., 2006) was applied so as to evaluate if the observed cluster distribution differs from random expectations. Principal coordinate analysis, applied using the UniFrac method to compute differences between microbial communities, showed that the data sets obtained from the mono-association samples cluster together, whereas data sets associated with bi- and multiple association samples cluster separately (Figure 2). This finding suggests that the overall effect of the monoassociation on the cecal microbiota composition is different from that caused by bi- and multiple associations. Mono-association seem to generate a relatively weak perturbation in the microbiota of the cecum and consequently cluster together with T0 mice samples. In contrast, co-association of two or multiple bifidobacterial strains causes a clear shift in the microbiota composition of the murine cecum microbiota as demonstrated by the clustering of bi- and multiple association samples away from the T0 samples (Figure 2). These observations suggest that functional inputs through molecular interactions are exploited by bifidobacteria not only among themselves but also with other members of the cecal microbiota as has previously been described for Bact. thetaiotaomicron in a much more simplified microbiota environment (Sonnenburg et al., 2006).

\section{Co-occurrence and co-exclusion of phylotypes of the} mice gut microbiota

Initial OTU-based cluster analysis indicated the existence of co-occurrence/co-exclusion of microbial taxa when a bifidobacterial strain is present in the cecum, and we therefore investigated if the gut microbiota follows community assembly rules. In order to evaluate taxa coexistence, we evaluated the Kendall tau rank correlation between the genus Bifidobacterium and the principal genera found in the samples (genera showing relative abundance $>1 \%$ in at least one sample was considered) (Figure 2). Interestingly, following the introduction of bifidobacteria, represented by the $\mathrm{T} 1$ and $\mathrm{T} 2$ time points, a positive correlation between the genus Bifidobacterium and particular members of the microbiota, such as Bacteroides, Odoribacter, AF12 and an unclassified member of Bacteroidales, was observed. In contrast, a negative correlation was found for unclassified members of Lachnospiraceae (representing $43 \%$ of the average microbiota composition in the analyzed murine samples) and Alistipes (Figure 2). These findings are fully consistent with the results obtained from the shotgun metagenomic and metabolomic analyses (see below). In fact, members of Bacteroides, Odoribacter and Bacteroidales taxa have been shown to have a fundamental role in polysaccharide metabolism and Short Chain Fatty Acid (SCFA) production in the gut environment (Goker et al., 2011; den Besten et al., 2013; Rakoff-Nahoum et al., 2014). In contrast, when supplementation of bifidobacteria to mice had been discontinued (represented by the T3 time point), it caused a switch from positive to a negative correlation between bifidobacteria and particular members of the murine microbiota, for example, unclassified members of Lachnospiraceae, Odoribacter, Bacteroides and unclassified members of Bacteroidales (Figure 2). In addition, the T4 time point data shows a correlation pattern similar to those of the T1 and T2 time points, reflecting that the microbiota returns to the composition it had before the intervention, following discontinuation of bifidobacterial supplementation (Supplementary Figure S6). In order to further detail changes in microbiota composition upon bifidobacterial administration, we explored 16S rRNA profiling results at OTU level. Such OTUs encompass sequences with 97\% identity, thus representing a putative 'phylotype', which is a physical entity that stands for one or a few closely related bacterial populations (Zhang et al., 2013). The identified OTUs eliciting a high level of modulation (in terms of relative abundance increase/decrease) following bifidobacterial supplementation correspond to those taxa identified by genus-level analysis (Supplementary Figures S6 and S7). Interestingly, a small number of OTUs (for example, OTU2352, OTU36184, OTU91120 and OTU3559) show a different modulatory behavior compared with the bacterial taxa to which they were assigned (Supplementary Figure S7), highlighting how individual phylotypes of the same genus can exhibit specific modulation patterns. As our knowledge regarding microbial taxa harboring the gastrointestinal tract is in many cases limited to general information about genera or higher taxonomic levels, evaluation of changes at genus level is usually exploited to provide an overview of the overall microbiota and its modulation. Nevertheless, analysis of results at OTU level represents a powerful tool to explore changes in microbiota composition at a much lower and informative level, thus allowing better understanding of its ecology and identification of key factors responsible for macro changes.

These observations are indicative of the modulatory responses of bifidobacterial administration on other murine gut commensals, and highlight the ability of the murine cecum microbiota to return to homeostasis following a perturbation, such as that due to the daily administration of a large number of bifidobacteria as performed in this study.

\section{Evaluation of the impact of bifidobacteria on the fecal} mice glycobiome

In order to evaluate the overall impact of bifidobacterial administration on the total gene content of the 
murine gut microbiota, we investigated the fecal microbiome for each murine group by means of shotgun metagenomic analyses of time points T0, T2 and T4. Evaluation of cluster orthologues gene functional family composition revealed the overall maintenance of a stable profile when bifidobacteria

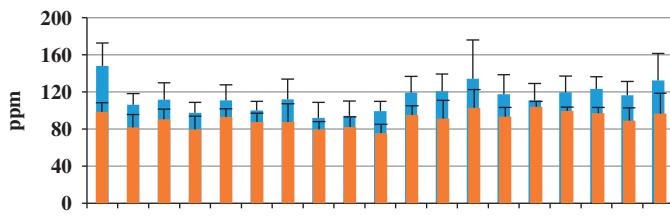

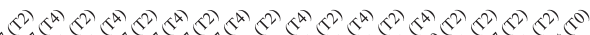
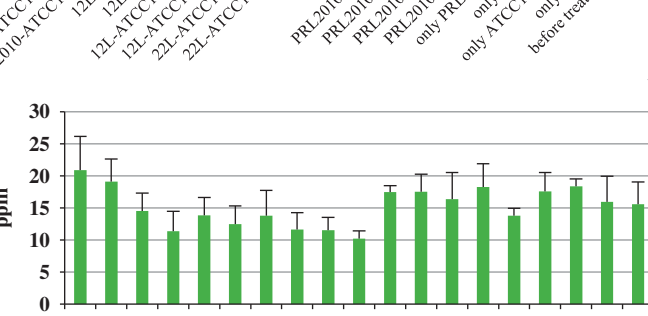

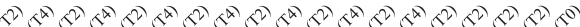

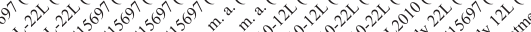

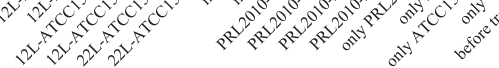

- Butyrate_R1

- Butyrate_R2

b
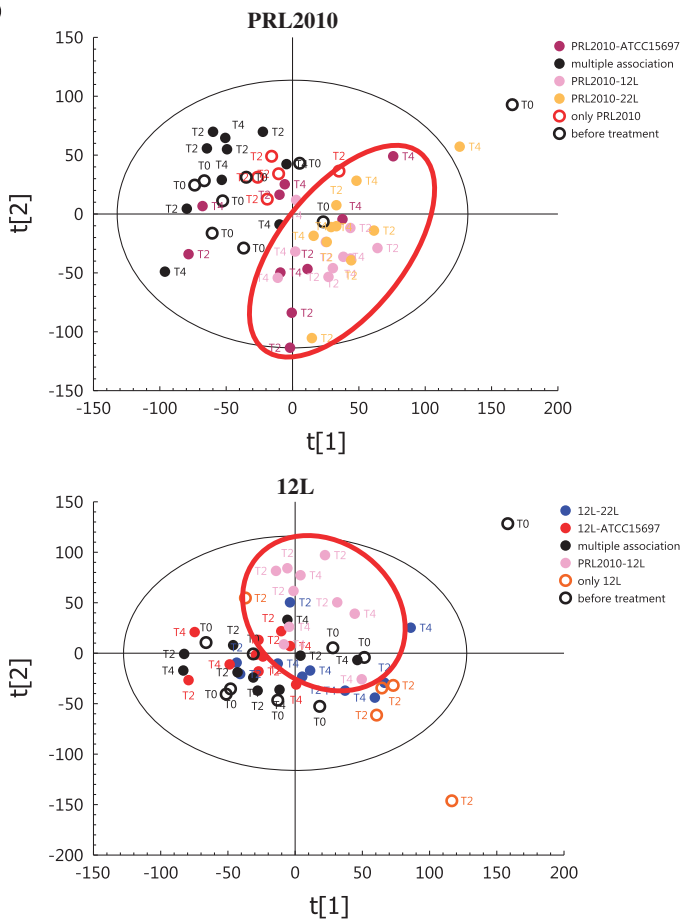

C

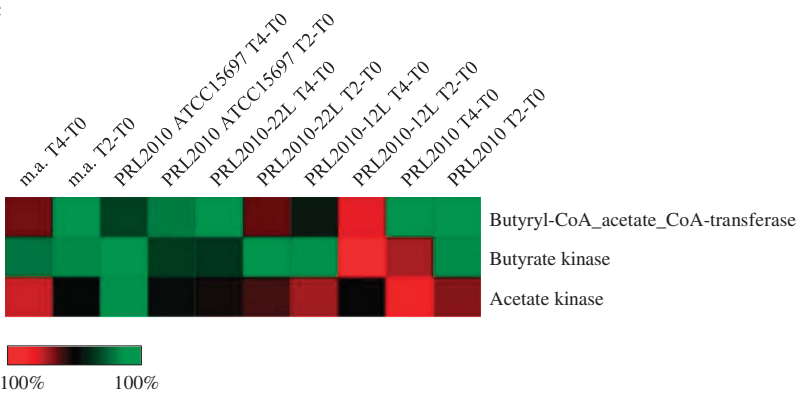

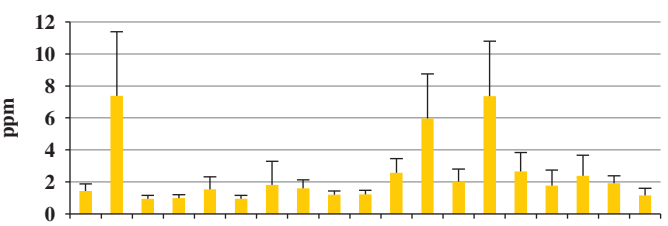

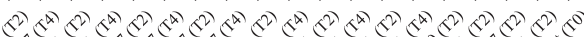

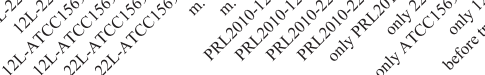

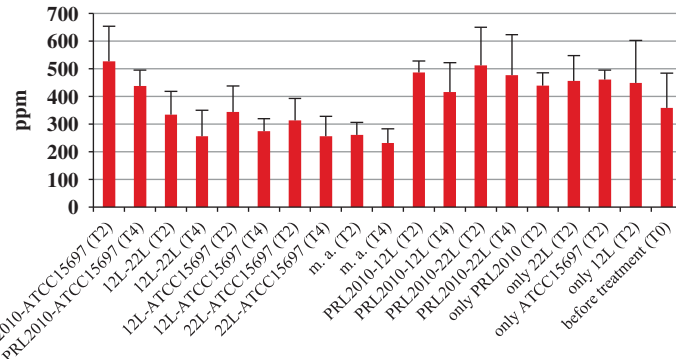

Formate $\quad$ Propionate Acetate
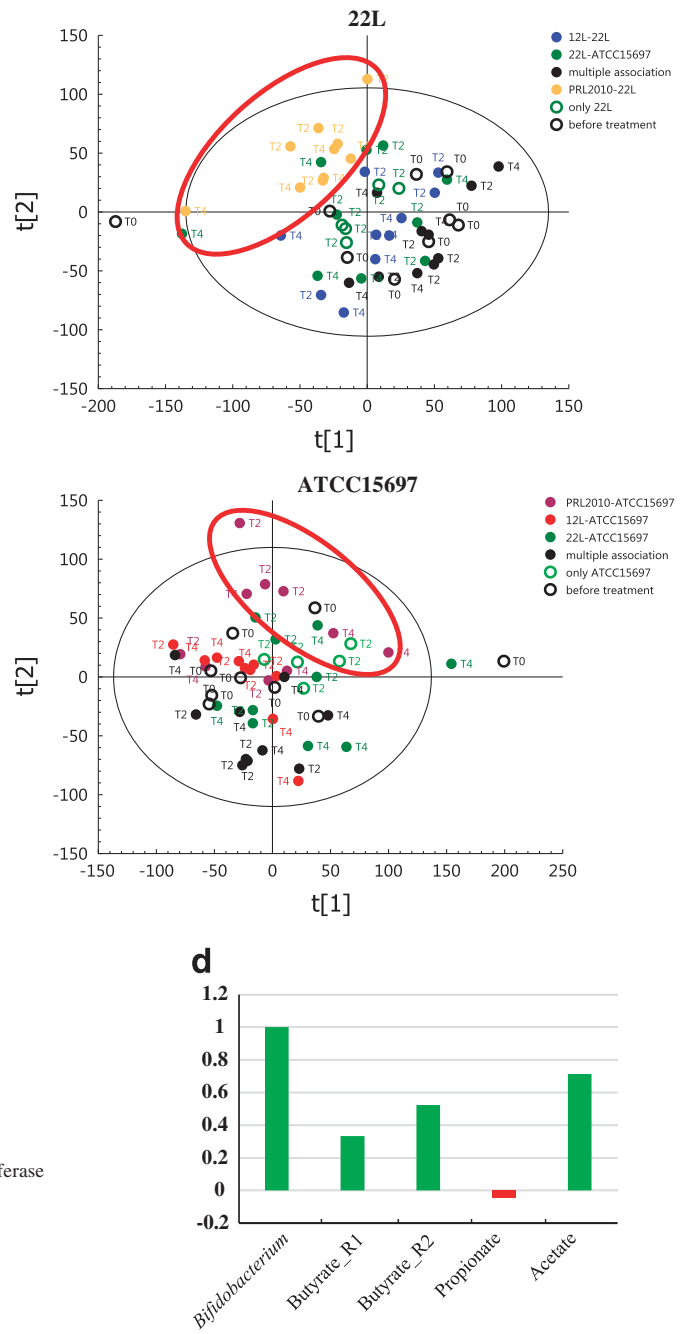
are supplemented to mice at time point T0 (Supplementary Figure S8). Interestingly, when the cluster orthologues gene profiles are clustered based on the three groups identified by $16 \mathrm{~S}$ rRNA profilebased hierarchical clustering (see above), groups B and $\mathrm{C}$ possess a somewhat larger gene repertoire involved in carbohydrate metabolism compared with group A $(P<0.001 ;$ Supplementary Figure S8). Screening of the metagenomic data sets for GHs allowed the identification of $22 \mathrm{GH}$ families whose relative abundance was shown to be increased following bifidobacterial administration (Supplementary Figure S8). Seventeen of these GH families exhibited an increased abundance in both mono- and bi-associations. These are predicted to be involved in the degradation of plant polysaccharides such as xylo-oligosaccharides, arabinoxylan, trehalose, mannose, as well as starch and starch-derivate. Notably, we also observed increased abundance of $\mathrm{GH} 20$, encompassing $\beta$-hexosaminidases, lacto- $N$ biosidases and $\beta-1,6-N$-acetylglucosaminidases, which are predicted to be involved in the degradation of host-glycans (Lombard et al., 2014). Interestingly, three of the remaining five $\mathrm{GH}$ families increase in their relative abundance, especially in bi-association samples. These GH families encompass genes encoding putative $\beta$ - $N$-acetylglucosaminidases (GH84), $\alpha$ $N$-acetylgalactosaminidases (GH109) and phosphorylases targeting galacto- $N$-biose and lacto- $N$-biose (GH112), all of which are directed toward hostglycan degradation (Lombard et al., 2014). In addition, the relative abundance of two $\mathrm{GH}$ families was significantly increased $(P<0.001)$ only in multiple association of $B$. bifidum PRL2010, $B$. breve 12L, $B$. adolescentis 22L and $B$. longum subsp. infantis ATCC15697. These two families specify presumed exo-glucosidases (GH15) and exo-mannosidases (GH92), which are both known to depolymerize polysaccharide chains (Supplementary Figure S8). Altogether, these shotgun metagenomic findings supported the notion that upon introduction of bifidobacteria in the cecum the corresponding murine glycobiome expands its enzymatic potential toward the degradation of plant-derived carbohydrates as well as host-glycan substrates. This is clearly consistent with the known saccharolytic features encoded by bifidobacteria (Turroni et al., 2010; Milani et al., 2014), as well as with the observed upregulation of bifidobacterial genes that are involved in the metabolism of polysaccharides under in vivo conditions (see above).

\section{Influence of bifidobacteria on the murine fecal metabolome}

As previously described, fecal metabolites correlate with gut microbiota composition (Claesson et al., 2012). We therefore performed metabolomic analysis of fecal extracts from the mice exposed to the various bifidobacterial strains (mono-, bi- and multiple associations). A representative metabolomic profile is presented in Figure 3, highlighting an evident trend of separation according to the community setting. Notably, the principal coordinate analysis representation based on all analyzed fecal metabolomic profiles show that mice that had been administered B. bifidum PRL2010 clearly cluster separately from the metabolomes obtained from the murine gut that had been associated with other bifidobacterial strains (Figure 3). This finding suggests a specific impact of PRL2010 on the host's metabolome. The major metabolites separating the various groups of mice were the SCFAs, including formate, acetate and propionate. SCFA are formed by microbial fermentation of dietary polysaccharides and they are associated with eliciting beneficial effects on the host (Wong et al., 2006). In this context, we observed a high level of formate and an increase in the level of acetate, as well as a low increase of propionate in murine cecal communities enriched by PRL2010 cells (Figure 3). The metabolic function of formate has not been well documented and its beneficial role, if any, is currently unknown. Nevertheless, formate has been described to be one of the main products of dietary polysaccharide fermentation by bifidobacteria especially under glycan-limiting conditions (Macfarlane and Macfarlane, 2003). The difference in formate production observed in PRL2010 associations may be due to the impact of B. bifidum PRL2010 toward a more saccharoclastic microbiota. In contrast, the levels of acetate and propionate were shown to decrease in the multi-association vs monoor bi-associations (Figure 3). This latter finding suggests that the modulation of the gut microbiota by multi-association of bifidobacteria promotes an enhancement of those enteric bacteria responsible for the utilization of acetate and propionate. In addition,

Figure 3 Impact of bifidobacterial administration on SCFA production by the murine microbiota. (a) Shows a bar plot representation of butyrate, propionate, acetate and formate concentrations, expressed in p.p.m., observed in murine fecal samples before and following bifidobacterial administration in mono-, bi- and multiple associations. (b) represents the principal coordinate analysis (PCoA) based on total NMR profiles of all analyzed fecal samples for each bifidobacterial strain/combination used in this study. Red circles highlight samples encompassing B. bifidum PRL2010. (c) displays a heat map representation showing the variation in percentage of the relative abundance of genes involved in butyrate and acetate production in samples encompassing $B$. bifidum PRL2010, observed through shotgun metagenomics analyses. (d) depicts a bar plot illustrating the covariance between the average bifidobacterial relative abundance resulting from the 16S rRNA profiling analyses and the average abundance of butyrate, propionate and acetate resulting from metabolomics analyses, in samples encompassing B. bifidum PRL2010 in mono-, bi- or multiple association. 
as acetate availability is linked to the production of butyrate (Louis and Flint, 2009), we investigated its metabolic production in all samples. As expected, a modest increase of this SCFA was identified only in mice that had been administered PRL2010 (Figure 3). Co-occurrence/co-exclusion analysis was performed between the bifidobacterial relative abundance of samples from mice administered B. bifidum in mono-, bi- or multiple associations and SCFAs concentrations. The results highlight positive covariance between the presence of $B$. bifidum in mono-, bi- or multiple associations, and production of acetate and butyrate by the gut microbiota, whereas production of propionate does not appear to be linked to $B$. bifidum exposure (Figure 3). In order to genetically support these findings, the fecal murine microbiomes were screened for the presence of genes predicted to encode acetate-kinases (Louis and Flint, 2009), or butyrate-kinases and butyryl-CoA:acetate-CoA-transferases (Louis and Flint, 2009), enzymes that are involved in acetate or butyrate synthesis, respectively. The obtained data are consistent with the metabolomic profiles and revealed an increase in genes dedicated to the production of butyrate in the murine fecal microbiomes enriched with $B$. bifidum PRL2010 (Figure 3). However, no variance in abundance of acetate kinase-encoding genes was observed, indicating that increased acetate production is linked to enhanced transcription of genes involved in acetate production and/or increased availability acetyl-phosphate or acetyl-CoA, which are the metabolic precursors of acetate (Louis and Flint, 2009; Figure 3). Additional metabolites that differed in concentration among the analyzed samples also encompass carbohydrates. As expected, upon supplementation of bifidobacteria in mono-, bi- and multiple association to mice, a significant shift $(P<0.001)$ was observed of the fecal metabolome with respect to simple glycans such as $\alpha$ - and $\beta$-glucose, as well as $\alpha$ - and $\beta$-galactose that may have been produced from the breakdown of complex diet-derived and/or hostderived polysaccharides (Supplementary Figure S9). This trend is particularly apparent for fecal samples from mice that had been administered $B$. bifidum PRL2010 on its own, from mice with bi-associations of $B$. bifidum PRL2010 with $B$. breve 12L or $B$. adolescentis 22L, and from mice associated with multiple bifidobacterial strains (Supplementary Figure S9), thus reinforcing the previous genomic observations of increased saccharolytic performance of these bifidobacterial strains with particular emphasis on the impact of B. bifidum PRL2010.

\section{Conclusions}

Co-occurrence of bifidobacterial strains in the murine cecum was shown to enhance the level of persistence of each strain compared with the mono-associations, thus suggesting the establishment of a mutualistic effect, which is directed to the harvest/utilization of glycans. Bifidobacteria clearly modulate their gene expression toward an increase in glycan metabolism, including both diet-derived polysaccharides, such as xylan, arabinoxylan, mannose and starch, as well as host-derived glycans (for example, mucin). These data are fully consistent with the predicted saccharolytic behavior of bifidobacteria (Milani et al., 2014), and indicate that bifidobacteria engage in a synergistic harvesting strategy toward enteric glycans because of the employment of (partly) complementary GH repertoires they encode (Supplementary Figure S10) in a similar manner to what was previously reported for enteric bacteria (Kato et al., 2005; Flint et al., 2007; El Aidy et al., 2013). Such metagenomic findings support the notion that bifidobacteria have an ecological role in shaping the gut microbiome toward an enrichment of saccharolytic microbiota members such as Bacteroidetes (Martens et al., 2009), where possible cross-feeding activities exert a key role. The transient presence of bifidobacteria also affects the overall composition of the murine gut microbiota, as was demonstrated when such data were compared between conditions of bifidobacterial multi- and mono-associations. Notably, the composition of this microbiota seems to return to its pre-administration status once bifidobacterial administration had been discontinued, thus following a similar trend to what had previously been reported for other microbiotadisturbing agents such as xenobiotics and antibiotics (Power et al., 2014). The interplay between bifidobacteria also appears to affect the overall metabolic profile of the gut microbiota underlying possible implications for the physiology of the host. In this context, bifidobacterial communities appear to influence SCFA production by the cecal microbiota, either directly by modulating SCFA synthesis, in particular acetate and formate (Macfarlane and Macfarlane, 2003), or indirectly by altering bacterial associations and/or microbe-microbe interactions (Macfarlane and Macfarlane, 2003). Acquiring knowledge on the intricate molecular relationships that occur between members of bifidobacterial communities residing in the mammalian gut are not only crucial in order to understand the genetic strategies followed by bifidobacteria to colonize and persist in the human intestine, but are also pivotal in order to appreciate and investigate the overall microbial dynamics within the mammalian gut.

\section{Conflict of Interest}

The authors declare no conflict of interest.

\section{Acknowledgements}

We thank GenProbio srl for financial support of the Laboratory of Probiogenomics. This work was financially supported by an IRCSET Embark postdoctoral fellowship and a FEMS Advance Fellowship to FT. DvS and FT are members of The APC Microbiome Institute funded by Science Foundation Ireland (SFI), through the Irish Government's National Development Plan (Grant number SFI/12/RC/2273). 


\section{References}

Barcenilla A, Pryde SE, Martin JC, Duncan SH, Stewart CS, Henderson C et al. (2000). Phylogenetic relationships of butyrate-producing bacteria from the human gut. Appl Environ Microbiol 66: 1654-1661.

Bottacini F, O'Connell Motherway M, Kuczynski J, O'Connell KJ, Serafini F, Duranti S et al. (2014). Comparative genomics of the Bifidobacterium breve taxon. BMC Genomics 15: 170.

Claesson MJ, Jeffery IB, Conde S, Power SE, O'Connor EM, Cusack S et al. (2012). Gut microbiota composition correlates with diet and health in the elderly. Nature 488: $178-184$.

De Vuyst L, Leroy F. (2011). Cross-feeding between bifidobacteria and butyrate-producing colon bacteria explains bifdobacterial competitiveness, butyrate production, and gas production. Int J Food Microbiol 149: 73-80.

den Besten G, van Eunen K, Groen AK, Venema K, Reijngoud DJ, Bakker BM. (2013). The role of shortchain fatty acids in the interplay between diet, gut microbiota, and host energy metabolism. J Lipid Res 54: 2325-2340.

Duncan SH, Flint HJ. (2008). Proposal of a neotype strain (A1-86) for Eubacterium rectale. Request for an opinion. Int J Syst Evol Microbiol 58: 1735-1736.

Duranti S, Turroni F, Lugli GA, Milani C, Viappiani A, Mangifesta M et al. (2014). Genomic characterization and transcriptional studies of the starch-utilizing strain Bifidobacterium adolescentis 22L. Appl Environ Microbiol 80: 6080-6090.

Egan M, Motherway MO, Kilcoyne M, Kane M, Joshi L, Ventura M et al. (2014a). Cross-feeding by Bifidobacterium breve UCC2003 during co-cultivation with Bifidobacterium bifidum PRL2010 in a mucinbased medium. BMC Microbiol 14: 282.

Egan M, O'Connell Motherway M, Ventura M, van Sinderen D. (2014b). Metabolism of sialic acid by Bifidobacterium breve UCC2003. Appl Environ Microbiol 80: 4414-4426.

El Aidy S, Van den Abbeele P, Van de Wiele T, Louis P, Kleerebezem M. (2013). Intestinal colonization: how key microbial players become established in this dynamic process Microbial metabolic activities and the interplay between the host and microbes. Bioessays 35: 913-923.

Falony G, Calmeyn T, Leroy F, De Vuyst L. (2009). Coculture fermentations of Bifidobacterium species and Bacteroides thetaiotaomicron reveal a mechanistic insight into the prebiotic effect of inulin-type fructans. Appl Environ Microbiol 75: 2312-2319.

Ferrario C, Milani C, Mancabelli L, Lugli GA, Turroni F, Duranti S et al. (2015). A genome-based identification approach for members of the genus Bifidobacterium. FEMS Microbiol Ecol 91: pii: fiv009.

Flint HJ, Duncan SH, Scott KP, Louis P. (2007). Interactions and competition within the microbial community of the human colon: links between diet and health. Environ Microbiol 9: 1101-1111.

Fondevila M, Dehority BA. (1994). Degradation and utilization of forage hemicellulose by rumen bacteria, singly in coculture or added sequentially. J Appl Bacteriol 77: 541-548.

Goker M, Gronow S, Zeytun A, Nolan M, Lucas S, Lapidus A et al. (2011). Complete genome sequence of Odoribacter splanchnicus type strain (1651/6). Stand Genomic Sci 4: 200-209.
Gueimonde M, Tolkko S, Korpimaki T, Salminen S. (2004). New real-time quantitative PCR procedure for quantification of bifidobacteria in human fecal samples. Appl Environ Microbiol 70: 4165-4169.

Kato S, Haruta S, Cui ZJ, Ishii M, Igarashi Y. (2005). Stable coexistence of five bacterial strains as a cellulosedegrading community. Appl Environ Microbiol 71: 7099-7106.

Lombard V, Golaconda Ramulu H, Drula E, Coutinho PM, Henrissat B. (2014). The carbohydrate-active enzymes database (CAZy) in 2013. Nucleic Acids Res 42: D490-D495.

Louis P, Flint HJ. (2009). Diversity, metabolism and microbial ecology of butyrate-producing bacteria from the human large intestine. FEMS Microbiol Lett 294: 1-8.

Lozupone C, Hamady M, Knight R. (2006). UniFrac-an online tool for comparing microbial community diversity in a phylogenetic context. BMC Bioinformatics 7: 371.

Macfarlane S, Bahrami B, Macfarlane GT. (2011). Mucosal biofilm communities in the human intestinal tract. Adv Appl Microbiol 75: 111-143.

Macfarlane S, Macfarlane GT. (2003). Regulation of shortchain fatty acid production. Proc Nutr Soc 62: 67-72.

Martens EC, Koropatkin NM, Smith TJ, Gordon JI. (2009). Complex glycan catabolism by the human gut microbiota: the Bacteroidetes Sus-like paradigm. J Biol Chem 284: 24673-24677.

Matsuki T, Watanabe K, Fujimoto J, Miyamoto Y, Takada T, Matsumoto K et al. (2002). Development of $16 \mathrm{~S}$ rRNA-gene-targeted group-specific primers for the detection and identification of predominant bacteria in human feces. Appl Environ Microbiol 68: 5445-5451.

Milani C, Lugli GA, Duranti S, Turroni F, Bottacini F, Mangifesta M et al. (2014). Genomic encyclopedia of type strains of the genus Bifidobacterium. Appl Environ Microbiol 80: 6290-6302.

Morrison DJ, Mackay WG, Edwards CA, Preston T, Dodson B, Weaver LT. (2006). Butyrate production from oligofructose fermentation by the human faecal flora: what is the contribution of extracellular acetate and lactate? Br J Nutr 96: 570-577.

Munoz-Tamayo R, Laroche B, Walter E, Dore J, Duncan SH, Flint HJ et al. (2011). Kinetic modelling of lactate utilization and butyrate production by key human colonic bacterial species. FEMS Microbiol Ecol 76: $615-624$.

O'Connell Motherway M, Zomer A, Leahy SC, Reunanen J, Bottacini F, Claesson MJ et al. (2011). Functional genome analysis of Bifidobacterium breve UCC2003 reveals type IVb tight adherence (Tad) pili as an essential and conserved host-colonization factor. Proc Natl Acad Sci USA 108: 11217-11222.

Poutanen K, Sundberg M, Korte H, Puls J. (1990). Deacetylation of xylans by acetyl esterases of Trichoderma-Reesei. Appl Microbiol Biot 33: 506-510.

Power SE, O'Toole PW, Stanton C, Ross RP, Fitzgerald GF. (2014). Intestinal microbiota, diet and health. Br J Nutr 111: 387-402.

Rakoff-Nahoum S, Coyne MJ, Comstock LE. (2014). An ecological network of polysaccharide utilization among human intestinal symbionts. Curr Biol 24: 40-49.

Rescigno M. (2014). Intestinal microbiota and its effects on the immune system. Cell Microbiol 16: 1004-1013. 
Reuter G. (1963). Comparative studies on the bifidus flora in the feces of infants and adults. With a contribution to classification and nomenclature of bifidus strains. Zentralbl Bakteriol Orig 191: 486-507.

Robles Alonso V, Guarner F. (2013). Linking the gut microbiota to human health. Br J Nutr 109(Suppl 2): S21-S26.

Sela DA, Chapman J, Adeuya A, Kim JH, Chen F, Whitehead TR et al. (2008). The genome sequence of Bifidobacterium longum subsp. infantis reveals adaptations for milk utilization within the infant microbiome. Proc Natl Acad Sci USA 105: 18964-18969.

Sonnenburg JL, Angenent LT, Gordon JI. (2004). Getting a grip on things: how do communities of bacterial symbionts become established in our intestine? Nat Immunol 5: 569-573.

Sonnenburg JL, Chen CT, Gordon JI. (2006). Genomic and metabolic studies of the impact of probiotics on a model gut symbiont and host. PLoS Biol 4: e413.

Tannock GW, Wilson CM, Loach D, Cook GM, Eason J, O'Toole PW et al. (2012). Resource partitioning in relation to cohabitation of Lactobacillus species in the mouse forestomach. ISME J 6: 927-938.

Turroni F, Bottacini F, Foroni E, Mulder I, Kim JH, Zomer A et al. (2010). Genome analysis of Bifidobacterium bifidum PRL2010 reveals metabolic pathways for hostderived glycan foraging. Proc Natl Acad Sci USA 107: 19514-19519.

Turroni F, Foroni E, Pizzetti P, Giubellini V, Ribbera A, Merusi P et al. (2009). Exploring the diversity of the bifidobacterial population in the human intestinal tract. Appl Environ Microbiol 75: 1534-1545.

Turroni F, Peano C, Pass DA, Foroni E, Severgnini M, Claesson MJ et al. (2012a). Diversity of bifido- bacteria within the infant gut microbiota. PLoS One 7: e36957.

Turroni F, Serafini F, Foroni E, Duranti S, O'Connell Motherway M, Taverniti V et al. (2013). Role of sortasedependent pili of Bifidobacterium bifidum PRL2010 in modulating bacterium-host interactions. Proc Natl Acad Sci USA 110: 11151-11156.

Turroni F, Strati F, Foroni E, Serafini F, Duranti S, van Sinderen D et al. (2012b). Analysis of predicted carbohydrate transport systems encoded by Bifidobacterium bifidum PRL2010. Appl Environ Microbiol 78: 5002-5012.

Turroni F, Taverniti V, Ruas-Madiedo P, Duranti S, Guglielmetti S, Lugli GA et al. (2014). Bifidobacterium bifidum PRL2010 modulates the host innate immune response. Appl Environ Microbiol 80: 730-740.

Ventura M, O'Flaherty S, Claesson MJ, Turroni F, Klaenhammer TR, van Sinderen D et al. (2009). Genome-scale analyses of health-promoting bacteria: probiogenomics. Nat Rev Microbiol 7: 61-71.

Ventura M, Turroni F, Motherway MO, MacSharry J, van Sinderen D. (2012). Host-microbe interactions that facilitate gut colonization by commensal bifidobacteria. Trends Microbiol 20: 467-476.

Wolfensohn S, Lloyd M. (2003). Handbook of Laboratory Animal Managment and Welfare. Blackwell: Oxford.

Wong JM, de Souza R, Kendall CW, Emam A, Jenkins DJ. (2006). Colonic health: fermentation and short chain fatty acids. J Clin Gastroenterol 40: 235-243.

Zhang C, Li S, Yang L, Huang P, Li W, Wang S et al. (2013). Structural modulation of gut microbiota in life-long calorie-restricted mice. Nat Commun 4: 2163.

Supplementary Information accompanies this paper on The ISME Journal website (http://www.nature.com/ismej) 